\title{
Development of Antioxidant Packaging Film Based on Chinese Bayberry Tannin Extract and Polyvinyl Alcohol
}

\author{
Jingjing Liao ${ }^{1,2}$, Jinxing $\mathrm{Li}^{2}$, Hongyan Wang ${ }^{3}$, Yan $\mathrm{Zhu}^{2}$, Hisham Essawy ${ }^{4}$, Guanben $\mathrm{Du}^{1,2, *}$ and \\ Xiaojian Zhou $^{2, *}$ \\ ${ }^{1}$ Beijing Forestry University, Beijing, 100083, China \\ ${ }^{2}$ Yunnan Provincial Key Laboratory of Wood Adhesives and Glued Products, Southwest Forestry University, Kunming, 650224, \\ China \\ ${ }^{3}$ Zhejiang Academy of Forestry, Hangzhou, 310023, China \\ ${ }^{4}$ Department of Polymers and Pigments, National Research Centre, Cairo, 12622, Egypt \\ *Corresponding Authors: Xiaojian Zhou. Email: xiaojianzhou@hotmail.com; Guanben Du. Email: gongben9@hotmail.com
}

Received: 10 February 2021 Accepted: 06 March 2021

\begin{abstract}
The current work explores the potential use of commercial Chinese bayberry tannin (BT) to develop antioxidant PVA-based films using solvent casting process for packaging applications. The effect of BT concentration on opacity, water resistance and antioxidant capacity of resulting films was investigated. Properties like tensile strength, thermal behavior, and morphological aspects were also characterized. The experimental results showed that PVA/BT films formed with uniformly brown color and generally good transparency, offering good antioxidant ability. The PVA film containing BT presented slightly higher water resistance according to the results of moisture content and water vapor permeability, especially at low BT content ( $<10 \mathrm{wt} \%)$. The PVA can be compounded with up to $10 \mathrm{wt} \%$ BT without any obvious deterioration in the tensile strength. The PVA/BT films exhibited better thermal degradation behavior compared with PVA alone because of the chemical bonds of PVA-BT and the formation of char at high temperature. Based on the results, PVA incorporated with Chinese bayberry tannin may provide broader formulation options for packaging materials with antioxidant action.
\end{abstract}

\section{KEYWORDS}

Polyvinyl alcohol film; Chinese bayberry tannin; antioxidant; packaging material

\section{Introduction}

Conventional packaging made from fossil resources has been widely used in various applications, the over-consumption of such nondegradable and non-recyclable packaging has caused serious environmental problems. Therefore, there is an increasing demand for green packaging made from bio-based resources to substitute petroleum-based packaging materials [1,2]. Nowadays, the main trend for packaging innovations is the development of active packaging having antioxidant, anti-UV, and antimicrobial activities, especially for food and biomedical applications for the purpose of effective protection of manufactured products from deterioration during transportation, storage and display steps $[3,4]$. 
Polyvinyl alcohol (PVA) is a water-soluble, nontoxic, biocompatible, and biodegradable linear synthetic polymer. The excellent film-forming character, acceptable mechanical and thermal properties make PVA quite suitable to design sustainable active packaging materials [1,2]. The incorporation of PVA with natural active ingredients, such as chitosan [4-6], essential oils [7], tomato by-products extract [8], and lignin $[9,10]$, were frequently reported recently for developing PVA-based films containing antioxidant and/or antimicrobial functions. Compared with synthetic active agents, the use of natural active ingredients limited the risk of potential toxicity by migration especially in the field of food packaging and biomedical applications.

Tannins are important secondary metabolites and are widely presented in plants. They are famous for their bio-physicochemical properties, including antioxidant, anti-ultraviolet (UV), antifungal and antimicrobial capacity [11]. Generally, the word "tannin" represents two classes of phenolic compounds based on their chemical natures: hydrolysable tannins and condensed tannins [12]. In recent years, tannins have been proved to reduce the impact of thermo-oxidative and UV degradation, extending the shelf life of polymeric materials [13-16], including PVA-based materials [17]. On this basis, tannins can be used to design PVA-based active packaging films. Tea extracts [6,18,19], gallic acid [20], quercetin [20], tannin acid [21], and tannins from wood or wood bark [17,22] were reported to incorporate with PVA, displaying good anti-oxidant and antimicrobial activity of the resulting films by releasing antioxidant agents when in use. Among the above mentioned tannins, condensed tannins are more attractive to develop active packaging materials because of the high availability (more than $90 \%$ of the worldwide production of commercial tannins) and a relative low price (especially for those extracted from wood barks) [12]. Since last century, the main applications of condensed tannins were dedicated for the adhesives industry as a source of phenolics and conventional uses like leather tanning [23]. However, they have not been commonly used in the preparation of active packaging materials.

In this work, various concentrations of condensed tannin extracted from Chinese bayberry (Myrica rubra Sieb. et Zucc.), which is an important economic plant widely grown in Southern China [24,25], was used to develop PVA-based packaging film with antioxidant capacity. PVA-Chinese bayberry tannin (BT) film was prepared by a simple casting method. The relevant important characteristics for packaging like optical properties, moisture content, water solubility, water vapor permeability, and antioxidant capacity were fully characterized. The mechanical, morphological, thermal, and chemical structure of the resulting films were also identified.

\section{Materials and Methods}

\subsection{Materials}

PVA, glutaraldehyde (50\% vol/vol) and ethanol were purchased from Sinopharm chemical reagent Co., Ltd., Shanghai, China. The PVA has a hydrolysis degree of $98 \%-99 \%$ and a degree of polymerization around $1750 \pm 50$. 1,1-diphenyl-2-picrylhydrazyl (DPPH, 96\%) was bought from Shanghai Macklin Biochemical Co., Ltd., China. Condensed tannins extracted from Chinese bayberry, mainly consisted of prodelphinidins structures [26], were donated by Guangxi Wuming Company, Nanning, China, with $72 \%$ purification. The average molecular weight of commercial Chinese bayberry tannin (BT) is $2292 \mathrm{Da}$ [25]. All other materials were analytical reagents and used without purification.

\subsection{Film Preparation}

$5 \mathrm{wt} \%$ PVA solution was prepared at $95^{\circ} \mathrm{C}$ under magnetic stirring for more than $4 \mathrm{~h}$ to ensure complete dissolution. PVA and BT were mixed in weight ratios of 100:0, 95:5, 90:10, 85:15,80:20 and 75:25, respectively. All mixtures were incorporated with $5 \%$ of glutaraldehyde as a crosslinker (based on the weight of PVA and BT), and constantly stirred for $10 \mathrm{~min}$ at room temperature until complete dissolution. Subsequently, the resulting homogeneous solutions were heated in a water bath at $80^{\circ} \mathrm{C}$ under magnetic 
stirring. After $30 \mathrm{~min}$, the obtained film forming solutions $(80 \mathrm{~mL})$ were casted on a self-made glass plate $(21 \mathrm{~cm} \times 21 \mathrm{~cm})$ and air-dried at room temperature $\left(15-20^{\circ} \mathrm{C}\right)$ under a relative moisture of $45-50 \%$. After $72 \mathrm{~h}$, the films were peeled off for further use. The resulting films were termed PVA, P5T, P10T, P15T, P20T, P25T based on BT content.

\subsection{Characterizations}

\subsubsection{Thickness}

The thickness of each film was determined using a micrometer with a resolution of $0.001 \mathrm{~mm}$ (Everte, Shenzhen, China). The thickness of each specimen was measured at 10 random positions and average thickness was calculated.

\subsubsection{Opacity Characteristics}

The opacity of films was determined by measuring the absorbance at a wavelength of $600 \mathrm{~nm}$ using UV spectrometer according to the method of Wen et al. [19]. Each specimen was cut into a rectangular strip with $4 \mathrm{~cm} \times 1 \mathrm{~cm}$ dimension and placed in a spectrophotometer cell. The opacity was evaluated using Eq. (1):

Opacity $=\frac{\operatorname{Abs}_{600}}{\mathrm{x}}$

where, $\mathrm{A}$ is the absorption of film at $600 \mathrm{~nm}, \mathrm{x}$ is the thickness of the film.

\subsubsection{Moisture Content}

Specimen sections of $2 \mathrm{~cm} \times 2 \mathrm{~cm}$ were prepared for moisture content test . All samples were preweighted $\left(\mathrm{M}_{0}\right)$ and dried in an oven at $105^{\circ} \mathrm{C}$ to a constant weight $\left(\mathrm{M}_{1}\right)$. The moisture content (MC) of the films was calculated using Eq. (2). Three replicates were performed for each measurement.

$\operatorname{MC}(\%)=\frac{\mathrm{M}_{0}-\mathrm{M}_{1}}{\mathrm{M}_{0}} \times 100$

\subsubsection{Water Solubility}

Each film was cut into $2 \mathrm{~cm} \times 2 \mathrm{~cm}$ sections and weighed $\left(\mathrm{M}_{0}\right)$. The specimens were placed in distilled water at room temperature for $24 \mathrm{~h}$. Then, the films were removed and dried to a constant weight at $105^{\circ} \mathrm{C}$ (M1). At least three replicates were performed for each measurement. The water solubility (WS) was calculated based on Eq. (3):

$\mathrm{WS}(\%)=\frac{\mathrm{M}_{0}-\mathrm{M}_{1}}{\mathrm{M}_{0}} \times 100$

\subsubsection{Water Vapor Permeability}

The water vapor permeability (WVP) was determined after the method described by Peng et al. [6] and Wen et al. [19] with same modifications. The films were cut into round pieces with $80 \mathrm{~mm}$ diameter and sealed into beakers (inner diameter: $50 \mathrm{~mm}$, height: $70 \mathrm{~mm}$ ) filled with $3 \mathrm{~g}$ granular anhydrous calcium chloride. Then, the covered bakers were placed in a desiccator containing $1 \mathrm{~L}$ distilled water, providing $\mathrm{RH}$ gradients of $100 \%$. The covered beakers were weighed every $12 \mathrm{~h}$ for 3 consecutive days. The WVP was calculated using Eq. (4):

$\mathrm{WVP}=\frac{\mathrm{W} \times \mathrm{x}}{\mathrm{A} \times \Delta \mathrm{P} \times \mathrm{t}}$ 
JRM, 2022, vol.10, no.1

where, $\mathrm{W}$ is the weight increment of the covered beaker $(\mathrm{g}), \mathrm{x}$ is the thickness of film $(\mathrm{mm}), \mathrm{t}$ is the duration (s) of the increased weight of the covered beaker, $\mathrm{A}$ is the permeation area of the film sample $\left(\mathrm{m}^{2}\right)$, and $\Delta \mathrm{P}$ is the partial vapor pressure $\left(18^{\circ} \mathrm{C}, 2063.79 \mathrm{~Pa}\right)$.

\subsubsection{Antioxidant Activity}

The antioxidant activity of the films was evaluated by DPPH radical scavenging activity (RSA) according to a previously reported method [18]. Each sample $(0.1 \mathrm{~g})$ was soaked into $30 \mathrm{~mL}$ distilled water under magnetic stirring for $30 \mathrm{~min}$. The obtained solution $(1 \mathrm{~mL})$ was withdrawn and mixed with $4 \mathrm{~mL}$ of DPPH methanol solution $(150 \mu \mathrm{mol} / \mathrm{L})$. The mixture was kept in dark for $30 \mathrm{~min}$. Then, the absorbance of the mixture was measured at $517 \mathrm{~nm}$ using a WFZUV-8802S ultraviolet spectrophotometer (Unico Shanghai Instrument Co., Ltd., Shanghai, China). Each assay was performed in triplicate. The DPPH radical scavenging activity was determined according to Eq. (5):

$R S A(\%)\left[1-\frac{\mathrm{A}_{\text {sample }}}{\mathrm{A}_{\text {control }}}\right] \times 100$

where, $\mathrm{A}_{\text {control }}$ is the absorbances of the control using $1 \mathrm{~mL}$ distilled water replacing the sample solution. $\mathrm{A}_{\text {sample }}$ is the absorbances of the tested sample.

\subsubsection{Releasing Tannin Content}

Each sample $(0.1 \mathrm{~g})$ was soaked into $30 \mathrm{~mL}$ distilled water under magnetic stirring for $30 \mathrm{~min}$. The absorbance of obtained solution was measured at $275 \mathrm{~nm}$ using WFZUV-8802S ultraviolet spectrophotometer (Unico Shanghai Instrument Co., Ltd., Shanghai, China). Three repetitions were tested for each sample. The average of absorbances at $275 \mathrm{~nm}(\mathrm{ABS}, \lambda=275 \mathrm{~nm})$ were calculated.

\subsubsection{Tensile Test}

The specimens for tensile testing were cut into rectangles of $160 \mathrm{~mm}$ in length and $20 \mathrm{~mm}$ in wide. The tensile test was performed at room temperature $\left(27^{\circ} \mathrm{C}\right)$ and a relative humidity of $46 \%$ on SUST universal testing machine (model 5569, China), operated according to GB/T 1040.3-2006. The cross-head speed was set at $50 \mathrm{~mm} / \mathrm{min}$. Five repetitions were tested for each sample.

\subsubsection{Morphology}

The cross-sectional micrographs of films were observed using scanning electron microscopy (SEM) (Zeiss Sigma 300, Germany) operated with an accelerating voltage of $20 \mathrm{kV}$. The samples were previously frozen in liquid nitrogen and fractured. The cross-sections were gold coated before observation.

\subsubsection{Thermal Stability}

The thermal behavior of the films was performed using a thermogravimetric analyzer (TGA) (TG209F1, Germany). The tested samples $(5-10 \mathrm{mg})$ were heated from 30 to $700^{\circ} \mathrm{C}$ under nitrogen protection with a constant heating rate of $10^{\circ} \mathrm{C} / \mathrm{min}$.

\subsubsection{Infrared Spectroscopy}

All films were characterized using a Thermo scientific Nicolet IS50 FT-IR spectrometer (China) in an attenuated total reflection mode, in the range from 4000 to $600 \mathrm{~cm}^{-1}$ at $4 \mathrm{~cm}^{-1}$ resolution for a total of 32 scans.

\section{Results and Discussion}

\subsection{Physical Appearance and Opacity}

Physical appearance and opacity of films are critical properties for packaging applications. The surface images and opacity of resulting films were presented in Fig. 1. The physical appearance (including color and 
transparency) of films can be directly observed from the surface images. All PVA/BT films showed uniform brown color with generally good transparency. However, their transparency decreased with the increase of BT content. Opacity value corresponds to a higher visible light absorbance of a sample at a given thickness and associates with the appearance and color of films [19]. As can be seen from Fig. 1, the opacity values of PVA/BT films are higher than that of the pure PVA film except P5T, indicating that high content of BT $(>5 \mathrm{wt} \%$ ) can decrease the transparency of films.

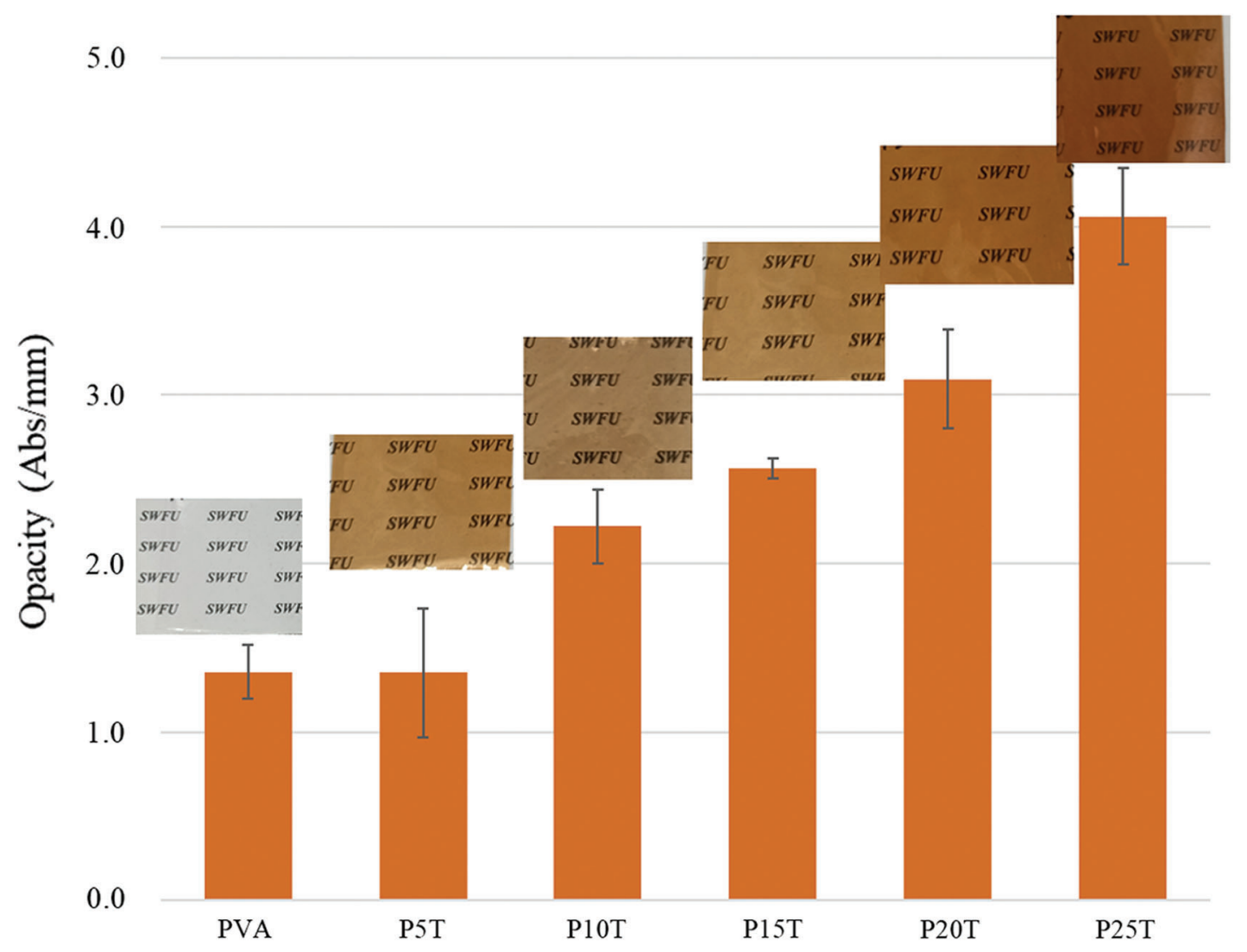

Figure 1: Opacity and digital images of PVA and PVA/BT films

\subsection{Thickness, Moisture Content, Water Solubility and Water Vapor Permeability}

The basic film properties, including thickness, moisture content (MC), water solubility (WS) and water vapor permeability (WVP) are presented in Tab. 1. The thickness of PVA/BT films slightly increased compared with PVA since the addition of BT slightly increased the concentration of film-forming solution. The MC represents the water resistance of films, especially in humid environment. The MC of films decreased with the addition of BT, which can be explained by reduction of hydroxyl groups that attract water to the surface of films, caused by crosslinking reactions in presence of glutaraldehyde together with developed hydrogen bonds between BT and PVA [22]. The PVA/BT films presented higher WS compared with PVA due to the release of less strongly bound BT when immersed in water. This can be confirmed by the typical UV absorbance value of BT at $275 \mathrm{~nm} \mathrm{[27]} \mathrm{as} \mathrm{shown} \mathrm{in} \mathrm{Fig.} \mathrm{2.} \mathrm{The} \mathrm{WVP}$ values of PVA/BT films (except P10T) generally increased with the addition of BT. This result could be related to the increase of free volume and chain mobility in the film network, enhancing the passage of water molecules across the film. One possible reason is the crosslinked structure makes the organization of PVA in crystalline lattice difficult [28]. Another possible reason could be the existing pores in the films containing high concentrations of the bulky BT, which can be observed below in the micrographs taken by electron microscopy. 
Table 1: Thickness, moisture content, water solubility and water vapor permeability of PVA/BT films

\begin{tabular}{lllll}
\hline Samples & Thickness $(\mathrm{mm})$ & MC $(\%)$ & WS $(\%)$ & WVP $\times 10^{-7}(\mathrm{~g} / \mathrm{m} \cdot \mathrm{s} \cdot \mathrm{Pa})$ \\
\hline PVA & $0.08 \pm 0.01$ & $11.38 \pm 0.19$ & $13.42 \pm 1.33$ & $1.12 \pm 0.06$ \\
P5T & $0.11 \pm 0.03$ & $10.43 \pm 0.60$ & $16.94 \pm 1.69$ & $1.19 \pm 0.08$ \\
P10T & $0.09 \pm 0.01$ & $10.87 \pm 0.86$ & $17.20 \pm 0.32$ & $1.00 \pm 0.15$ \\
P15T & $0.10 \pm 0.00$ & $10.72 \pm 0.73$ & $17.85 \pm 0.43$ & $1.57 \pm 0.02$ \\
P20T & $0.09 \pm 0.01$ & $10.07 \pm 0.54$ & $21.12 \pm 0.54$ & $1.40 \pm 0.02$ \\
P25T & $0.10 \pm 0.00$ & $10.81 \pm 0.35$ & $21.58 \pm 0.27$ & $1.28 \pm 0.10$ \\
\hline
\end{tabular}

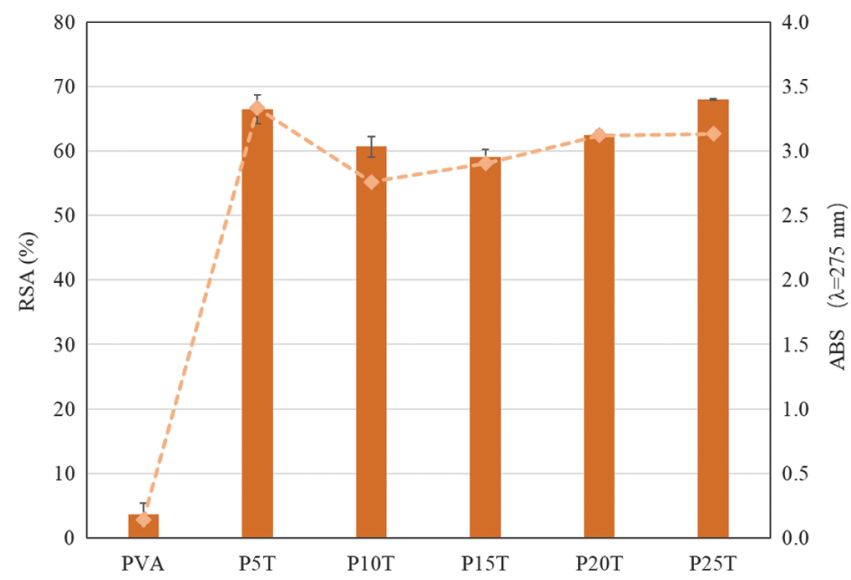

Figure 2: DPPH radical scavenging activity (column) and UV absorbance at $275 \mathrm{~nm}$ (line)

\subsection{Antioxidant Activity}

The antioxidant activity of the PVA/BT films was evaluated through DPPH assay, which is considered one of the most standardized methods for antioxidant capacity evaluation [29]. The antioxidant capacity of PVA/BT films depends on the release of BT in solution, consuming the DPPH free radicals. The release of BT in solution was determined by the typical UV absorbance value of BT at $275 \mathrm{~nm}$ [27].

As can be found in Fig. 2, the PVA film without BT presented slightly DPPH radical scavenging activity ( $\sim 6 \%)$ as reported by Luo [30], owing to the presence of hydrogen on the carbon atoms with hydroxyl groups. With the addition of BT, the DPPH scavenging activity sharply increased from $6 \%$ to $60 \%$, and nearly to $70 \%$ in the case of P5T and P25T. This behavior associates with the release of BT from PVA matrix as confirmed by UV absorbance at $275 \mathrm{~nm}$ (Fig. 2). The main mechanism was that the resulting films swelled as water molecules penetrate the inner structure of polymer chains, loosening the network structure of the films. With the decrease of constraining force of the PVA matrix, free BT will be easily extracted into the solution, thereby increasing the DPPH radical scavenging capacity. According to the study of Luzi et al. [20], the release of antioxidant agents from PVA can be correlated with their concentrations in the films in the same period as can be observed an increasing trend of DPPH radical scavenging activity from PVA/BT films. The unexpected high value from P5T might be explained by the relatively low hydrogen and/or covalent bonds between PVA and BT at this relatively low concentration of tannin and glutaraldehyde. The DPPH radical scavenging activity did not increase by multiple as the concentration of BT increasing from $5 \mathrm{wt} \%$ to $25 \mathrm{wt} \%$, which is in agreement with the study of Luzi et al [20]. One possible reason is the presence of intensive hydrogen and/or covalent bonds between PVA and BT, decreasing the release rate of BT from PVA. Therefore, resulting PVA/BT films bearing slowreleasing capacity. In general, BT can therefore be an active agent for packaging materials preparation. 


\subsection{Tensile Properties}

Typical strain-stress curves of PVA and PVA/BT films are exhibited in Fig. 3 and their related data are included in Tab. 2. BT has great contribution to the Young's modulus of films, in agreement with PVA incorporated with rigid polyphenols [20]. The PVA-based films containing BT at low concentration have a limited influence on tensile strength and elongation at break. However, such properties were substantially reduced when compared with PVA. As the films were formed under mild conditions, the crosslinking bonds induced by glutaraldehyde in PVA-PVA and/or PVA-BT of obtained films were limited. Therefore, the excessive BT present in PVA matrix decreased the interaction force of PVA molecular chains, reducing tensile strength and elongation at break. Besides, excessive amount of BT might enhance their self-interaction during the film preparation, producing discontinuing film structure. This can be proved by the presented dots and pores in P20T (Fig. 4).

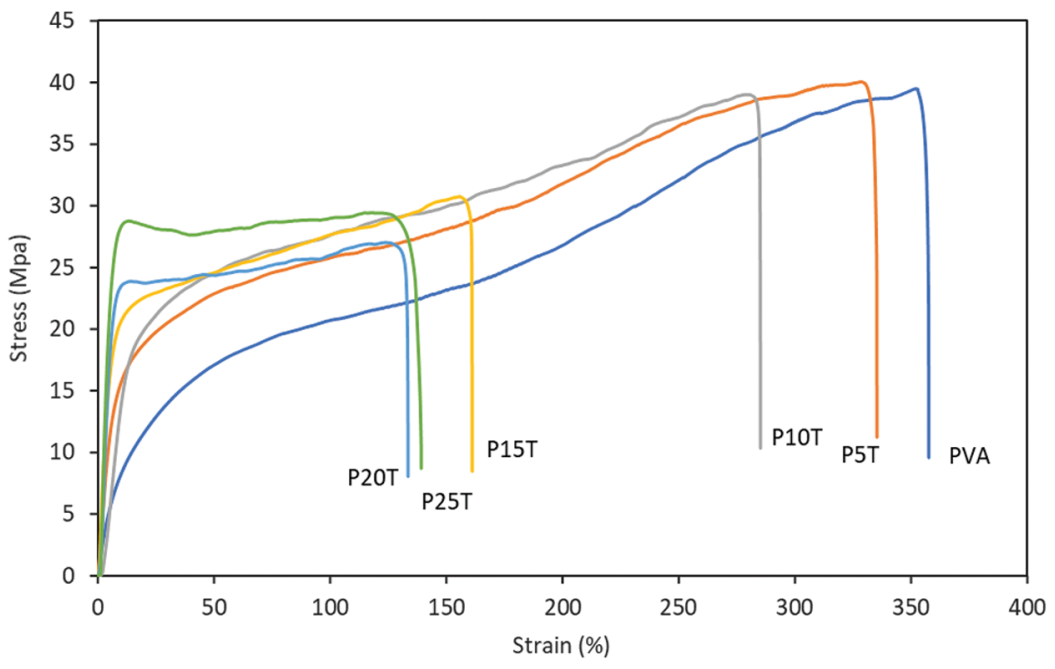

Figure 3: Stress-strain curves of PVA and PVA/BT films

Table 2: Mechanical parameters of PVA and PVA/BT films

\begin{tabular}{llll}
\hline Samples & Young's modulus (MPa) & Tensile strength (MPa) & Elongation at break (\%) \\
\hline PVA & $17.5 \pm 3.7$ & $39.0 \pm 5.2$ & $353.5 \pm 38.22$ \\
P5T & $69.4 \pm 16.3$ & $40.0 \pm 3.7$ & $327.0 \pm 42.9$ \\
P10T & $64.7 \pm 20.6$ & $38.7 \pm 2.1$ & $282.8 \pm 18.7$ \\
P15T & $282.8 \pm 40.8$ & $30.7 \pm 2.2$ & $153.5 \pm 24.4$ \\
P20T & $503.8 \pm 65.3$ & $30.7 \pm 1.0$ & $134.5 \pm 9.9$ \\
P25T & $570.2 \pm 42.9$ & $27.0 \pm 2.2$ & $123.1 \pm 43.3$ \\
\hline
\end{tabular}

\subsection{Morphology}

The morphology of PVA incorporated with different BT contents was investigated using SEM imaging. cross-sectional micro-structures of PVA, P10T, and P20T fractured surfaces at two different magnifications are shown in Fig. 4. In case of PVA, it reveals a homogeneous, smooth and uniform fractured surface. This phenomenon highlights the homogeneity of PVA during solvent casting process [20,31]. On the other hand, the morphology of P10T and P20T revealed less smooth compared with PVA. Further, slight coarse domains 
were easily found in the fractured surface (marked with white circles), especially when increasing the concentration of BT to $20 \mathrm{wt} \%$. This illustrates that the addition of the rigid BT reduced the ductility of the films, especially after the crosslinking and hydrogen bonding [32]. It should be noted that the fractured surface of $\mathrm{P} 20 \mathrm{~T}$ also presented some dots and pores probably caused by the agglomeration of BT during the solvent casting process, producing discontinuing film structure. This result was also observed in PVA incorporated with high concentration of tea polyphenol [33].
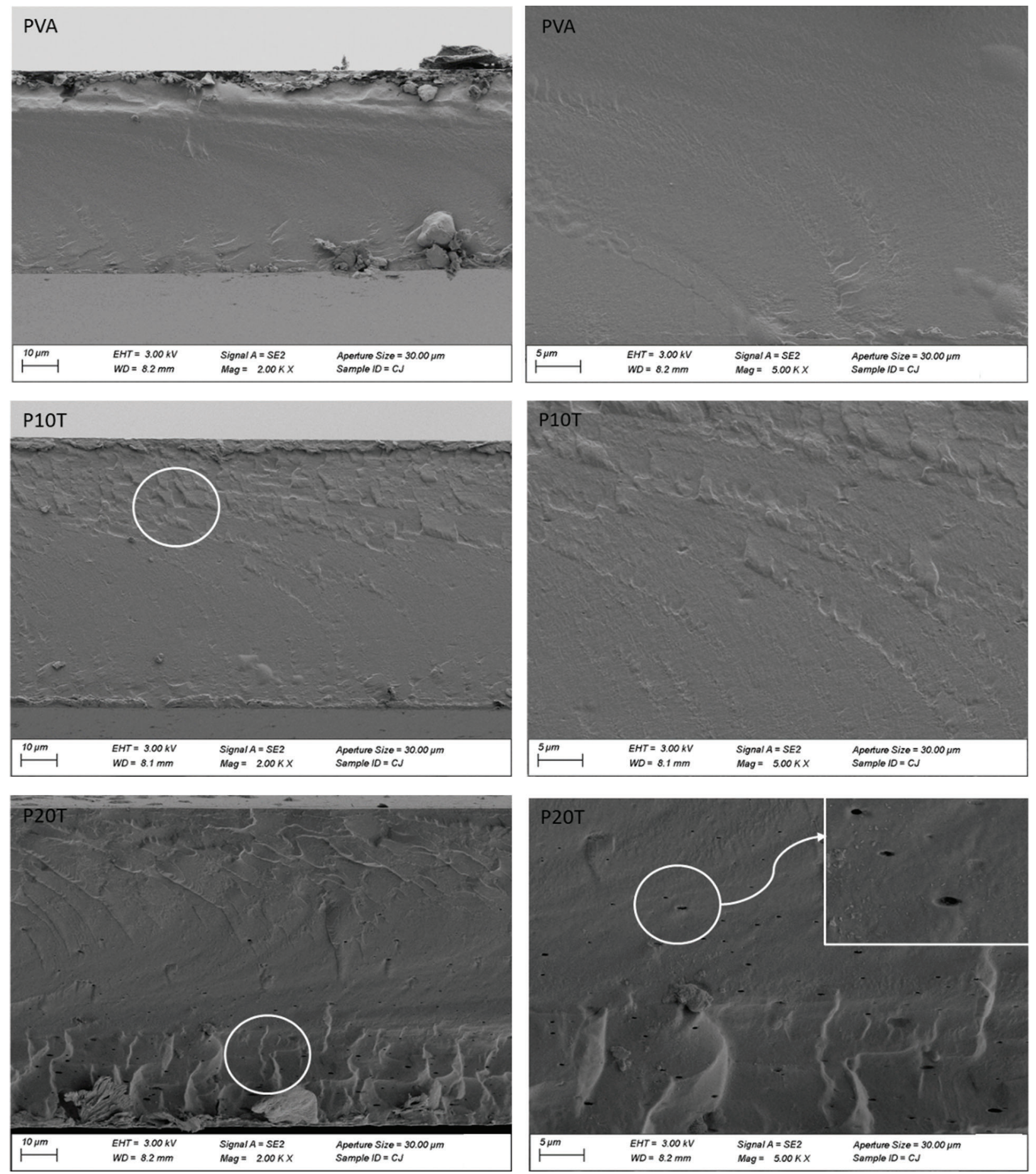

Figure 4: SEM micrographs of fractured cross-sections for selected films at two magnifications 


\subsection{Thermal Stability}

For investigating the effect of BT loading on the thermal degradation behavior of PVA-based films, thermal analysis was performed and TG curves were recorded in Fig. 5. All samples presented three consecutive degradation steps. The first stage $\left(40\right.$ to $\left.200^{\circ} \mathrm{C}\right)$ is mainly related to the release of water and volatile content, the main intermolecular hydrogen bonding cleavage of PVA and the decomposition of linkages between BT units [34]. The second stage $\left(200\right.$ to $\left.350^{\circ} \mathrm{C}\right)$ is corresponding to the breakage of the main chains. The third step $\left(350\right.$ to $\left.600^{\circ} \mathrm{C}\right)$ illustrates the cleavage of the $\mathrm{C}-\mathrm{C}$ backbone of the PVA and the production of carbon molecules and hydrocarbons [28]. As can be seen from Fig. 5, the main difference in thermal behaviors between PVA and PVA/BT films is the last two stages. The residual mass and the initial decomposing temperature of PVA/BT films in the second stage are higher with respect to that of PVA, indicating BT postponed the decomposition of the main polymer chains in PVA [35] because of the hydrogen and/or covalent bonds between PVA and BT. In the final step, the residual weight of samples increases with increasing BT content due to the high char forming capacity of BT. This result is generally typical in polymeric composites containing phenolic compounds $[10,36]$.

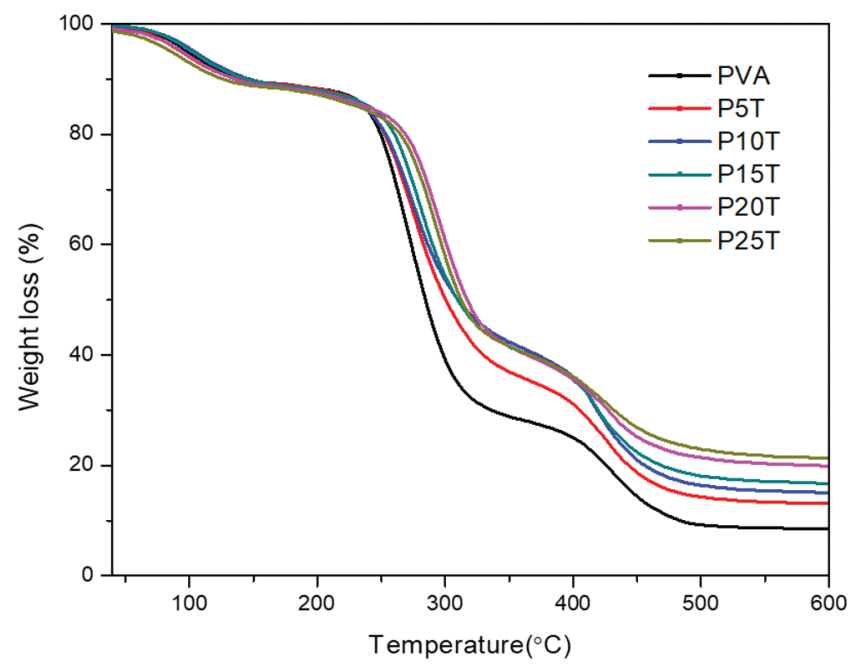

Figure 5: Thermal degradation traces of PVA and PVA/BT films

\subsection{FTIR Analysis}

The chemical interactions in the resulting films were investigated using FTIR spectroscopy Fig. 6 demonstrates FT-IR spectra of BT, PVA, P10T, and P20T. In case of BT, a broad band centered at $3400 \mathrm{~cm}^{-1}$ is ascribed to $-\mathrm{OH}$ stretching vibrations. The peaks at $1610 \mathrm{~cm}^{-1}$ and $1450 \mathrm{~cm}^{-1}$ are related to $\mathrm{C}=\mathrm{C}$ stretching and $\mathrm{C}-\mathrm{H}$ bending, respectively. The peak at $1340 \mathrm{~cm}^{-1}$ is attributed to $-\mathrm{OH}$ stretching of phenolic hydroxyl while that at $1050 \mathrm{~cm}^{-1}$ referred to C-O-C stretching of ether groups [18,37]. The PVA shows typical intense peaks: a broad band centered at $3210 \mathrm{~cm}^{-1}$ is attributed to the hydroxyl group stretching, and peak at $1090 \mathrm{~cm}^{-1}$ is associated to C-O stretching of the PVA backbone. As compared with the spectrum of PVA without glutaraldehyde (supporting information), the change of band at $2920 \mathrm{~cm}^{-1}$ is ascribed to the asymmetric stretching of $\mathrm{CH}_{2}$ because of the introduction of $-\mathrm{O}-\mathrm{CH}_{2}-\mathrm{O}-$ in the crosslinked structure [38]. Based on the given FT-IR spectra, a peak corresponding to BT (P20T, marked with arrow) appeared with increasing BT content $1610 \mathrm{~cm}^{-1}$. This one together with the multiplicity found in the C-O region confirm the insertion of tannin into the backbone of PVA, implying that the linkage happened mainly to PVA because of its high molecular weight [28], which can be more evidenced by the preservation of the $\mathrm{OH}$ region unchanged in shape or position. 
JRM, 2022, vol.10, no.1

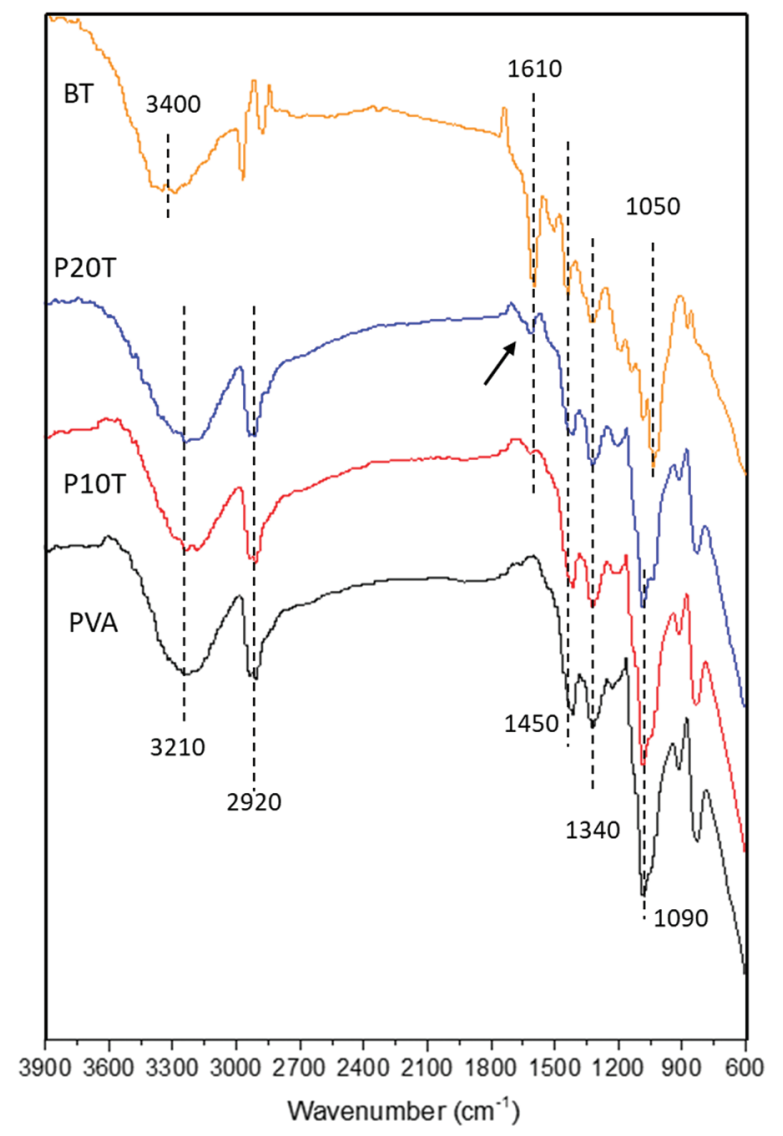

Figure 6: FTIR spectra of BT, PVA, and PVA/BT films

\section{Conclusion}

Condensed BT extracted from Chinese barberry can be introduced to PVA at various concentrations to develop antioxidant PVA-based films using a simple casting method. The DPPH free radical scavenging assays showed that PVA/BT films can present good antioxidant ability. The formed PVA/BT films can show uniform brown color and generally good transparency, especially when loaded with low BT content. The PVA films containing BT acquired relatively good water resistance according to moisture content and water vapor permeability, especially at low BT content, which additionally reveals the hydrophobicity of BT and the potential use of these materials for packaging applications. The addition of BT did not cause obvious changes in the mechanical properties when PVA is incorporated at low BT content $(<10 \mathrm{wt} \%)$. The SEM images revealed a homogeneous and uniform fractured surface of PVA/BT films indicating a good compatibility of PVA-BT, especially in PVA films bearing low BT content. The BT postponed the thermal degradation of PVA, due to the emerged chemical bonds of PVA-BT and the formation of char at higher temperatures. The interaction of PVA and BT in presence of tiny glutaraldehyde amount is limited most likely due to the high molecular weight of PVA thus the crosslinking occurred mainly within PVA polymer chains as revealed by FT-IR.

Funding Statement: This work was supported by the National Natural Science Foundation of China (NSFC 31971595, 31760187), the Program for Leading Talents of Science and Technology (Grant No. 2017HA013), the Yunnan Provincial Reserve Talents for Middle \& Young Academic and Technical Leaders (2019HB026), and the 111 Project. 
Conflicts of Interest: The authors declare that they have no conflicts of interest to report regarding the present study.

\section{References}

1. Aslam, M., Kalyar, M. A., Raza, Z. A. (2018). Polyvinyl alcohol: a review of research status and use of polyvinyl alcohol based nanocomposites. Polymer Engineering \& Science, 58(12), 2119-2132. DOI 10.1002/pen.24855.

2. Halima, N. B. (2016). Poly(vinyl alcohol): review of its promising applications and insights into biodegradation. RSC Advances, 6(46), 39823-39832. DOI 10.1039/C6RA05742J.

3. Cazón, P., Vázquez, M., Velazquez, G. (2018). Cellulose-glycerol-polyvinyl alcohol composite films for food packaging: evaluation of water adsorption, mechanical properties, light-barrier properties and transparency. Carbohydrate Polymers, 195(21), 432-443. DOI 10.1016/j.carbpol.2018.04.120.

4. Haghighi, H., Leugoue, S. K., Pfeifer, F., Siesler, H. W., Licciardello, F. et al. (2020). Development of antimicrobial films based on chitosan-polyvinyl alcohol blend enriched with ethyl lauroyl arginate (LAE) for food packaging applications. Food Hydrocolloids, 100(3), 105419. DOI 10.1016/j.foodhyd.2019.105419.

5. Balasubramaniam, M. P., Murugan, P., Chenthamara, D., Ramakrishnan, S. G., Salim, A. et al. (2020). Synthesis of chitosan-ferulic acid conjugated poly(vinyl alcohol) polymer film for an improved wound healing. Materials Today Communications, 25, 101510. DOI 10.1016/j.mtcomm.2020.101510.

6. Peng, Y., Wu, Y., Li, Y. (2013). Development of tea extracts and chitosan composite films for active packaging materials. International Journal of Biological Macromolecules, 59, 282-289. DOI 10.1016/ j.ijbiomac.2013.04.019.

7. Debiagi, F., Kobayashi, R. K. T., Nakazato, G., Panagio, L. A., Mali, S. (2014). Biodegradable active packaging based on cassava bagasse, polyvinyl alcohol and essential oils. Industrial Crops and Products, 52, 664-670. DOI 10.1016/j.indcrop.2013.11.032.

8. Szabo, K., Teleky, B. E., Mitrea, L., Călinoiu, L. F., Martău, G. et al. (2020). Active packaging-poly(vinyl alcohol) films enriched with tomato by-products extract. Coatings, 10(2), 141. DOI 10.3390/coatings10020141.

9. El-Nemr, K. F., Mohamed, H. R., Ali, M. A., Fathy, R. M., Dhmees, A. S. (2020). Polyvinyl alcohol/gelatin irradiated blends filled by lignin as green filler for antimicrobial packaging materials. International Journal of Environmental Analytical Chemistry, 100(14), 1578-1602. DOI 10.1080/03067319.2019.1657108.

10. Yang, W., Owczarek, J. S., Fortunati, E., Kozanecki, M., Mazzaglia, A. et al. (2016). Antioxidant and antibacterial lignin nanoparticles in polyvinyl alcohol/chitosan films for active packaging. Industrial Crops and Products, 94(2), 800-811. DOI 10.1016/j.indcrop.2016.09.061.

11. Quideau, S., Deffieux, D., Douat-Casassus, C., Pouysegu, L. (2011). Plant polyphenols: chemical properties, biological activities, and synthesis. Angewandte Chemie International Edition, 50(3), 586-621. DOI 10.1002/ anie. 201000044 .

12. Arbenz, A., Avérous, L. (2015). Chemical modification of tannins to elaborate aromatic biobased macromolecular architectures. Green Chemistry, 17(5), 2626-2646. DOI 10.1039/C5GC00282F.

13. Bridson, J. H., Kaur, J., Zhang, Z., Donaldson, L., Fernyhough, A. (2015). Polymeric flavonoids processed with co-polymers as UV and thermal stabilisers for polyethylene films. Polymer Degradation and Stability, 122, 18-24. DOI 10.1016/j.polymdegradstab.2015.10.002.

14. Liao, J., Brosse, N., Pizzi, A., Hoppe, S. (2019). Dynamically cross-linked tannin as a reinforcement of polypropylene and UV protection properties. Polymers, 11(1), 102. DOI 10.3390/polym11010102.

15. Liao, J., Brosse, N., Pizzi, A., Hoppe, S., Xi, X. et al. (2019). Polypropylene blend with polyphenols through dynamic vulcanization: mechanical, rheological, crystalline, thermal, and UV protective property. Polymers, 11(7), 1108. DOI 10.3390/polym11071108.

16. Shnawa, H. A. (2017). Thermal stabilization of polyvinyl chloride with traditional and naturally derived antioxidant and thermal stabilizer synthesized from tannins. Journal of Thermal Analysis and Calorimetry, 129(2), 789-799. DOI 10.1007/s10973-017-6238-z. 
17. Zhai, Y., Wang, J., Wang, H., Song, T., Hu, W. et al. (2018). Preparation and characterization of antioxidative and UV-protective larch bark tannin/PVA composite membranes. Molecules, 23(8), 2073. DOI 10.3390/ molecules23082073.

18. Chen, C. W., Tang, Z. P., Ma, Y., Qiu, W. Q., Yang, F. X. et al. (2018). Physicochemical, microstructural, antioxidant and antimicrobial properties of active packaging films based on poly(vinyl alcohol)/clay nanocomposite incorporated with tea polyphenols. Progress in Organic Coatings, 123(2), 176-184. DOI 10.1016/j.porgcoat.2018.07.001.

19. Wen, H., Hsu, Y. I., Asoh, T. A., Uyama, H. (2020). Antioxidant activity and physical properties of pH-sensitive biocomposite using poly(vinyl alcohol) incorporated with green tea extract. Polymer Degradation and Stability, 178(14), 109215. DOI 10.1016/j.polymdegradstab.2020.109215.

20. Luzi, F., Pannucci, E., Santi, L., Kenny, J. M., Torre, L. et al. (2019). Gallic acid and quercetin as intelligent and active ingredients in poly(vinyl alcohol) films for food packaging. Polymers, 11(12), 1999. DOI 10.3390/ polym11121999.

21. Dai, H., Huang, Y., Huang, H. (2018). Enhanced performances of polyvinyl alcohol films by introducing tannic acid and pineapple peel-derived cellulose nanocrystals. Cellulose, 25(8), 4623-4637. DOI 10.1007/s10570-0181873-5.

22. da Cruz, J. A., da Silva, A. B., Ramin, B. B. S., Souza, P. R., Popat, K. C. et al. (2020). Poly(vinyl alcohol)/ cationic tannin blend films with antioxidant and antimicrobial activities. Materials Science and Engineering: $C$, 107, 110357. DOI 10.1016/j.msec.2019.110357.

23. Pizzi, A. (2019). Tannins: prospectives and actual industrial applications. Biomolecules, 9(8), 344. DOI 10.3390/ biom9080344.

24. Shen, J., Xie, L., Zhai, M., Lin, Q. (2004). Antiviral activity and chemical compositions of extracts from Myrica rubra. Journal of Fujian Agriculture and Forestry University (Natural Science Edition), 33, 441-443.

25. Teng, B., Jian, X., Gao, Y., Chen, W. (2016). Comparison of polyflavonoids in bayberry tanning effluent and commercial bayberry tannin: prerequisite information for vegetable tanning effluent recycling. Journal of Cleaner Production, 112, 972-979. DOI 10.1016/j.jclepro.2015.09.005.

26. Fu, Y., Qiao, L., Cao, Y., Zhou, X., Liu, Y. et al. (2014). Structural Elucidation and Antioxidant Activities of Proanthocyanidins from Chinese Bayberry (Myrica rubra Sieb. et Zucc.) Leaves. PLoS One, 9(5), e96162. DOI 10.1371/journal.pone.0096162.

27. Bensalah, N., Chair, K., Bedoui, A. (2018). Efficient degradation of tannic acid in water by $\mathrm{UV} / \mathrm{H}_{2} \mathrm{O}_{2}$ process. Sustainable Environment Research, 28(1), 1-11. DOI 10.1016/j.serj.2017.04.004.

28. Figueiredo, K. C. S., Alves, T. L. M., Borges, C. P. (2009). Poly(vinyl alcohol) films crosslinked by glutaraldehyde under mild conditions. Journal of Applied Polymer Science, 111(6), 3074-3080. DOI 10.1002/app.29263.

29. Floegel, A., Kim, D. O., Chung, S. J., Koo, S. I., Chun, O. K. (2011). Comparison of ABTS/DPPH assays to measure antioxidant capacity in popular antioxidant-rich US foods. Journal of Food Composition and Analysis, 24(7), 1043-1048. DOI 10.1016/j.jfca.2011.01.008.

30. Luo, J., Zuo, D., Deng, Z., Ji, A., Xia, G. (2020). Effects of heat treatment and tea polyphenols on the structure and properties of polyvinyl alcohol nanofiber films for food packaging. Coatings, 10(1), 49. DOI 10.3390/ coatings 10010049.

31. Luzi, F., Torre, L., Puglia, D. (2020). Antioxidant packaging films based on ethylene vinyl alcohol copolymer (EVOH) and caffeic acid. Molecules, 25(17), 3953. DOI 10.3390/molecules25173953.

32. Yang, W., Qi, G., Kenny, J. M., Puglia, D., Ma, P. (2020). Effect of cellulose nanocrystals and lignin nanoparticles on mechanical, antioxidant and water vapour barrier properties of glutaraldehyde crosslinked PVA films. Polymers, 12(6), 1364. DOI 10.3390/polym12061364.

33. Zhang, L., Shen, Q. (2020). Fully Green poly(vinyl alcohol)/tea polyphenol composites and super anti-ultraviolet and -bacterial properties. Macromolecular Materials and Engineering, 305(3), 1900669. DOI 10.1002/ mame.201900669.

34. Liao, J., Brosse, N., Hoppe, S., Du, G., Zhou, X. et al. (2020). One-step compatibilization of poly(lactic acid) and tannin via reactive extrusion. Materials \& Design, 191, 108603. DOI 10.1016/j.matdes.2020.108603. 
JRM, 2022, vol.10, no.1

35. Liu, Y., Wang, S., Lan, W. (2018). Fabrication of antibacterial chitosan-PVA blended film using electrospray technique for food packaging applications. International Journal of Biological Macromolecules, 107, 848-854. DOI 10.1016/j.ijbiomac.2017.09.044.

36. Liao, J., Brosse, N., Pizzi, A., Hoppe, S., Zhou, X. et al. (2020). Characterization and 3D printability of poly (lactic acid)/acetylated tannin composites. Industrial Crops and Products, 149, 112320. DOI 10.1016/j. indcrop.2020.112320.

37. García, D. E., Glasser, W. G., Pizzi, A., Osorio-Madrazo, A., Laborie, M. P. (2013). Hydroxypropyl tannin derivatives from Pinus pinaster (Ait.) bark. Industrial Crops and Products, 49, 730-739. DOI 10.1016/j. indcrop.2013.06.019.

38. Xue, B., Li, R., Deng, J., Zhang, J. (2016). Sound absorption properties of microporous poly(vinyl formal) foams prepared by a two-step acetalization method. Industrial \& Engineering Chemistry Research, 55(14), 3982-3989. 